\title{
Tuning of Nonlinear PID Controller for TRMS Using Evolutionary Computation Methods
}

\author{
J. SIVADASAN, M. WILLJUICE IRUTHAYARAJAN
}

\begin{abstract}
In this paper, the Twin rotor MIMO system (TRMS) is tuned by Nonlinear PID controller using Evolutionary Computation methods. The proposed Nonlinear PID controller, used to tune TRMS, improves the system performance with additional degrees of freedom. Evolutionary Computation methods such as Differential Search Algorithm (DSA), real coded Genetic Algorithm (RGA) with simulated binary crossover (SBX) and Particle Swarm optimization (PSO) and Gravitational Search Algorithm (GSA) are used to determine the optimal parameters of the proposed controller by minimizing Integral Square Error (ISE) for rotor response of TRMS. SIMULINK MATLAB software is used for simulating the system. The statistical performance of the controller is analysed among twenty independent trials by taking best, worst, mean and standard deviations of ISE. Simulation results reveal that TRMS system tuned by nonlinear PID controller using Particle Swarm optimization (PSO) is better than the other methods
\end{abstract}

Keywords: Evolutionary Computation; Nonlinear Controller; Twin Rotor System

\section{INTRODUCTION}

TRMS is a complex model of MIMO system, that resembles the behaviour of helicopter with unstable, nonlinear and cross coupled dynamics. So, tuning of TRMS is required and this can be done either by a linear or a nonlinear PID controller.

Proportional-Integral-Derivative (PID) control is easy and efficient in real time problems. Since 1942, with the advent of Ziegler - Nichols tuning method, the importance of PID control has developed significantly. Most of the industrial controllers adapt PID control algorithms, as no other controllers match the functionality, applicability and ease of use offered by the PID controllers [1]. Ziegler-Nichols and Cohen-Coon are the normally used conventional methods for tuning PID controllers. Neural networks, fuzzy based methods, neuro-fuzzy approach and evolutionary computation techniques are the advanced methods [2].

In recent decades, designing controllers for MIMO systems has engrossed a lot of research interests and many multivariable control approaches have been projected [3-5]. Various researchers have already reported the optimal design of PID controller for MIMO system using Evolutionary Computation (EC) methods like Genetic Algorithm [6, 7], Particle Swarm Optimization [8], Covariance Matrix Adaptation evolution strategy [9]. Later, Differential Search Algorithm (DSA) is developed for the solution of optimization problems. DSA simulates the Brownian-like random-walk movement used by an organism to migrate [10]. Also Gravitational Search Algorithm (GSA) is one of the evolutionary algorithms, proposed by Rashedi et al. [11]. It is utilized to find optimal location of Distributed Generation [12] and to design passive power filters for industrial power Systems [13]

Tuning the PID controller for MIMO systems using EC such as Differential Search Algorithm (DSA), real coded Genetic Algorithm (RGA) with simulated binary crossover (SBX), Particle Swarm optimization (PSO) and Gravitational Search Algorithm (GSA) are taken in this work.

Some researchers have reported about the Nonlinear PID controller structure [14]. Utilizing Nonlinear PID controller for tuning Linear Piezoelectric motors \& Superconducting Magnetic Energy Storage have been discussed $[15,16]$ and their research reveals that Nonlinear PID controller shows better performance than conventional linear PID controller. But the nonlinear constants used in the above research findings are fixed and not optimized [14-16]. Therefore in our study, tuning of Nonlinear PID controller for MIMO systems is considered and EC methods are used to optimize the nonlinear PID controller parameters.

Tao et al. proposed a fuzzy controller for TRMS [17] and in his work the minimization of error is not reported. Taskin [18] has proposed a fuzzy and LQR model with PID control. In his work the minimization of mean squared error is considered. Juang.et.al [19] proposed a modified fuzzy PID using RGA and reported the vertical and horizontal error. Later GA-PID control in FPGA was developed [20]. In their work they have compared the total error for vertical and horizontal angle separately for PID, GA-PID, RGA-PID, CMAC controller and GA-PID control in FPGA. In addition, real time adaptive nonlinear model inversion control [21] and Quasi LPV Statefeedback control was proposed [22]. Then fuzzy controller using Evolutionary algorithms was analysed [23] and they reported the comparison of average error obtained from Particle Swarm Optimization, Ant Bee Colony and Differential Evolution. Recently alternative methods tune the fractional order PID control for TRMS are reported $[24,25]$.

This paper focuses mainly on tuning of Nonlinear PID controller using EC for TRMS by minimizing the ISE of rotor. The remaining part of the paper is organized as follows. Section 2 introduces the description of TRMS. In Section 3 the parameters of Nonlinear PID controller and its simulation model is presented. Section 4 describes the tuning of Nonlinear PID controller for TRMS using Evolutionary computation. Section 5 describes the results and discussion. Finally, the results inferred are discussed in Section 6.

\section{TRMS}

TRMS is a complex model of MIMO system, its behaviour resembles that of a helicopter. As shown in Fig. 1, the TRMS mechanical unit consists of two rotors placed on a beam together with a counterbalance whose arm with weight at its end is fixed to the beam at the pivot and it determines a stable equilibrium position. The beam 
is hinged on its base in such a way that it can rotate freely both in the horizontal and vertical planes. Any one of the horizontal or the vertical degree of freedom can be restricted to 1 degree of freedom using nylon screws found near pivot point. At the two ends of the beam there are rotors (the main and tail rotors) driven by de motors. The main rotor develops a lifting force allowing the beam to rise vertically making a rotation around the pitch axis. Whereas, the tail rotor is used to make the beam turn left or right around the yaw axis. The whole unit is attached to the tower ensuring safe helicopter control experiments. Apart from the mechanical units, the electrical unit (located under the tower) plays a vital role for TRMS control. It allows for measured signals to be transmitted to the PC and control signal application through an I/O card. The mechanical and electrical units provide a complete control system setup. This device is a multivariable, nonlinear and sturdily coupled system, with degrees of freedom on the pitch and yaw angle denoted by $\psi$ and $\varphi$, correspondingly.

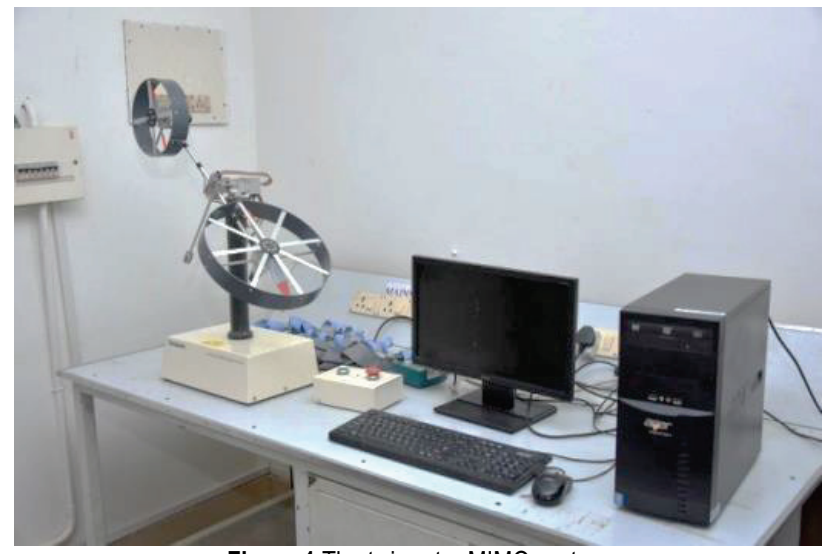

Figure 1 The twin rotor MIMO system

The state of the beam is explained by four process variables: horizontal and vertical angles measured by position sensors tied at the pivot, and two corresponding angular velocities. Two extra state variables are the momentum of the dc motors. In a typical helicopter, the aerodynamic force is controlled by varying the angle of attacker.

The laboratory setup is shown in Fig. 1 which is so constructed that the angle of attack is fixed. The aerodynamic force is controlled by adjusting the speed of the rotors. Therefore, the control inputs are the supply voltage of the dc motors. A change in the voltage value results in a change in the rotation speed of the propeller [26].

\section{NONLINEAR PID CONTROLLER}

The Nonlinear PID controller model proposed by Tan et.al [14] is utilized in our work. The nonlinear combination of the error signal is given by

$u(t)=k_{p} \cdot F(e, \alpha, \delta)+k_{i} \int e \mathrm{~d} t+\frac{\mathrm{d}}{\mathrm{d} t}[F(e, \alpha, \beta)]$

Where $k_{p}, k_{i}, k_{d}$ are PID parameters and $F(e, \alpha, \delta)$ is a nonlinear function, e is the position error, $\alpha$ and $\delta$ are the nonlinear constants

$F(e, \alpha, \delta)=\left\{\begin{array}{cc}|e|^{\alpha} \operatorname{sgn}(e), & |e|>\delta \\ \frac{e}{\delta^{1-\alpha}}, & |e| \leq \delta\end{array}\right.$

Empirically the design parameters are selected accordingly $\delta=0,1$ to $0,2, \alpha=0,5$ to 1,0 . The block diagram of the conventional nonlinear PID controller is shown in Fig. 2, it consists of two PID controllers, one for main and the other for the tail rotor. The conventional controller tunes the errors of main and tail rotor individually, cross coupled dynamics is not considered.

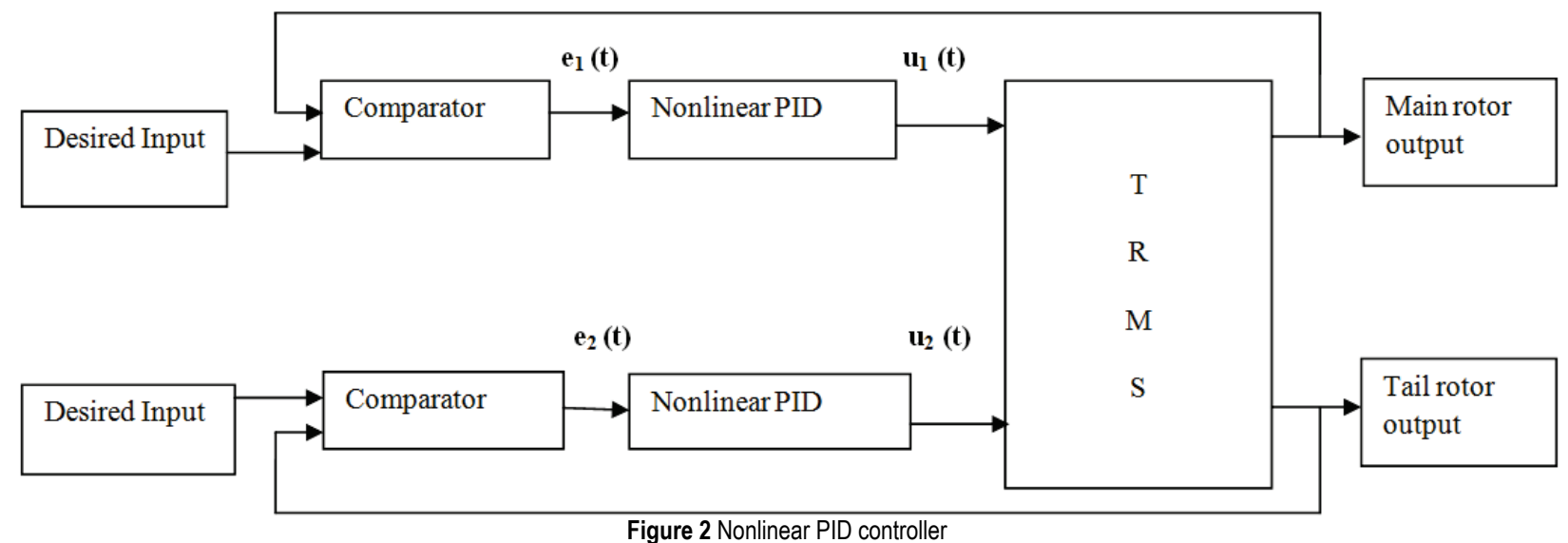

So, considering the cross coupled dynamics, the Cross coupled Nonlinear PID controller is developed as shown in Fig. 3, it comprises four PID controllers. The influence of main rotor in tail rotor and vice versa were considered for controlling the TRMS. 


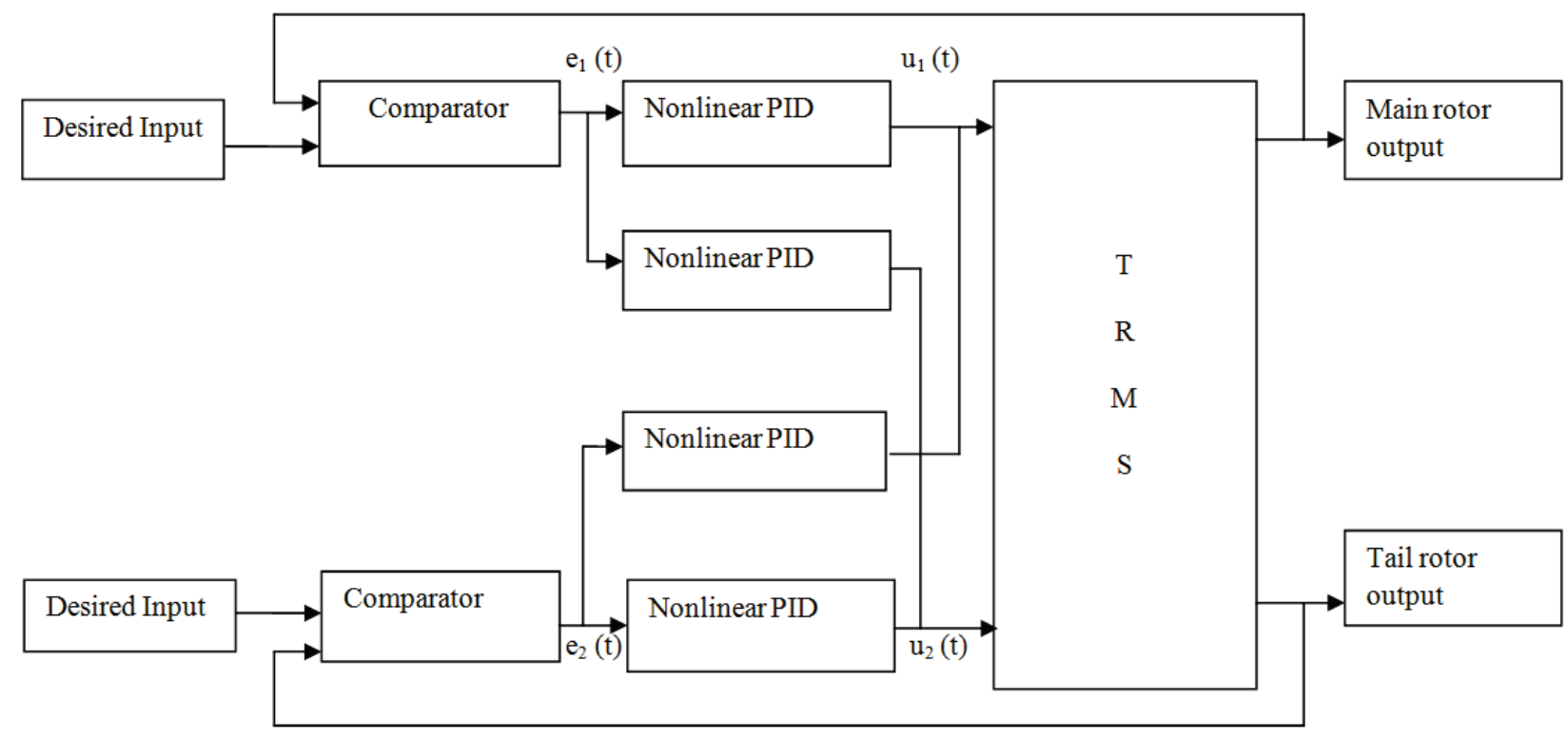

Figure 3 Cross coupled Nonlinear PID controller

\section{EC IMPLEMENTATION OF NONLINEAR PID CONTROLLER}

To optimize the PID parameters and nonlinear constants, Evolutionary algorithms are applied. For implementing them four cases have been considered: case(i) Nonlinear PID controller with fixed $\delta$ and $\alpha$ values, case(ii) Nonlinear PID controller with optimized $\delta$ and $\alpha$ values, case (iii) Cross coupled Nonlinear PID controller with fixed $\delta$ and $\alpha$ values, case(iv) Cross coupled Nonlinear PID controller with optimized $\delta$ and $\alpha$ values. In order to obtain the optimum performance, the parameters of controllers of main and tail rotors are given by

for case (i)

$$
K(s)=\left[K_{p_{r}} K_{i_{r}} K_{d_{r}} K_{p_{t}} K_{i_{t}} K_{d_{t}}\right]
$$

for case (ii)

$$
K(s)=\left[K_{p_{r}} K_{i_{r}} K_{d_{r}} K_{p_{t}} K_{i_{t}} K_{d_{t}} \delta \alpha\right]
$$

for case (iii)

$K(s)=\left[\begin{array}{c}K_{p_{r}} K_{i_{r}} K_{d_{r}} K_{p_{t r}} K_{i_{t r}} K_{d_{t r}} K_{p_{t}} \\ K_{i_{t}} K_{d_{t}} K_{p_{r t}} K_{i_{r t}} K_{d_{r t}}\end{array}\right]$

for case (iv)

$$
K(s)=\left[\begin{array}{c}
K_{p_{r}} K_{i_{r}} K_{d_{r}} K_{p_{t r}} K_{i_{t r}} K_{d_{t r}} K_{p_{t}} K_{i_{t}} \\
K_{d_{t}} K_{p_{r t}} K_{i_{r t}} K_{d_{r t}} \delta \alpha
\end{array}\right]
$$

where $K_{p_{r}}, K_{i_{r}}, K_{d_{r}}$ are PID parameters for main rotor, $K_{p_{t}}, K_{i_{t}}, K_{d_{t}}$ for tail rotor, $K_{p_{t r}}, K_{i_{t r}}, K_{d_{t r}}$ are parameters for influence of tail rotor on main rotor and
$K_{p_{r t}}, K_{i_{r t}}, K_{d_{r t}}$ are parameters for influence of main rotor on tail rotor

All the elements of chromosomes of the population are randomly initiated within the search space precised by their lower and upper bounds of individual parameters as follows

for case (i)

$0 \leq\left[K_{p_{r}} K_{i_{r}} K_{d_{r}} K_{p_{t}} K_{i_{t}} K_{d_{t}}\right] \leq 5$

$\delta=0,15$ and $\alpha=0,5$

for case (ii)

$0 \leq\left[K_{p_{r}} K_{i_{r}} K_{d_{r}} K_{p_{t}} K_{i_{t}} K_{d_{t}}\right] \leq 5$

$0,1 \leq \delta \leq 0,2$ and $0 \leq \alpha \leq 1$

for case (iii)

$0 \leq\left[\begin{array}{c}K_{p_{r}} K_{i_{r}} K_{d_{r}} K_{p_{t r}} K_{i_{t r}} K_{d_{t r}} K_{p_{t}} \\ K_{i_{t}} K_{d_{t}} K_{p_{r t}} K_{i_{r t}} K_{d_{r t}}\end{array}\right] \leq 5$

$\delta=0,15$ and $\alpha=0,5$

for case (iv)

$0 \leq\left[\begin{array}{c}K_{p_{r}} K_{i_{r}} K_{d_{r}} K_{p_{t r}} K_{i_{t r}} K_{d_{t r}} K_{p_{t}} \\ K_{i_{t}} K_{d_{t}} K_{p_{r t}} K_{i_{r t}} K_{d_{r t}}\end{array}\right] \leq 5$

$0,1 \leq \delta \leq 0,2$ and $0 \leq \alpha \leq 1$

The lower and upper bounds are chosen based on the literature. In this work, performance of Evolutionary computation within this chosen range has been considered. In future adaptive parameter range may be chosen. The population size is fixed at 30 for TRMS. The commonly used time-integral performance index, integral of the square error (ISE) is considered as objective in this paper 


$$
I S E=\int_{0}^{\infty} e_{1}^{2} \mathrm{~d} t+\int_{0}^{\infty} e_{2}^{2} \mathrm{~d} t
$$

where $e_{1}(t)$ is the error signal for main position rotor (elevation or pitch) and $e_{2}(t)$ is the error signal for tail position rotor (azimuth or yaw).

\section{RESULTS AND DISCUSSION}

For simulating TRMS, MATLAB SIMULINK software is employed. Simulations are carried out using core 2 Duo processor $3 \mathrm{GHz}, 4 \mathrm{~GB}$ RAM PC. ISE is determined for desired pitch /yaw angle response of set point regulation. Simulations are carried out using stopping criteria, maximum number of function evaluation (Feval) is set at 1500. Owing to randomness, many trials with independent population initializations are made to acquire a useful conclusion of the performance of the algorithm. The sampling time is $0,01 \mathrm{sec}$. Hence, best, mean, worst, standard deviations of ISE measure in 20 independent trials are reported. The simulation results are given as follows.

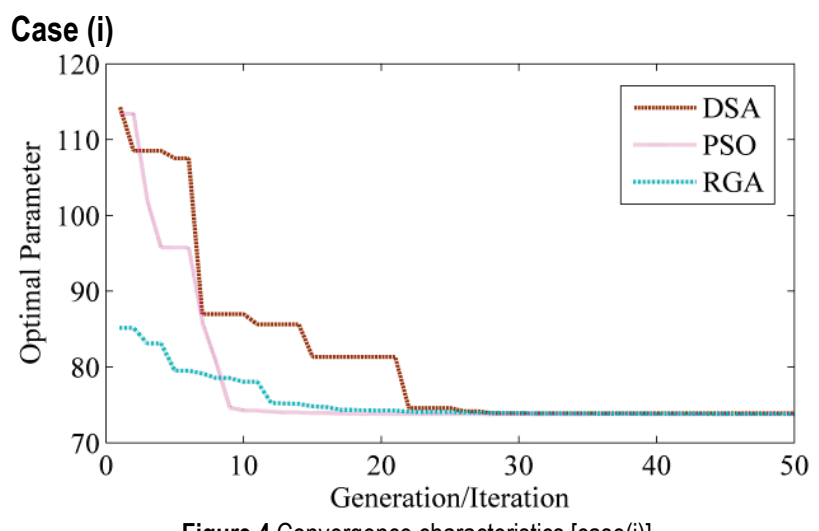

Figure 4 Convergence characteristics [case(i)]

Table 1 Statistical performance of DSA, PSO and RGA for the Nonlinear PID

\begin{tabular}{|c|c|c|c|}
\hline \multicolumn{4}{|c|}{ controller [case(i)] } \\
\hline Evolutionary Algorithm & DSA & PSO & RGA \\
\hline Best_optimal & 73,8880 & 73,7812 & 73,7864 \\
\hline & 3,0613 & 2,9922 & 2,9909 \\
& 5,0000 & 5,0000 & 4,9953 \\
Best_optimal_parameter & 5,0000 & 5,0000 & 4,9994 \\
& 5,0000 & 5,0000 & 5,0000 \\
& 5,0000 & 5,0000 & 4,9998 \\
& 1,2293 & 1,262 & 1,2666 \\
\hline Worst_foptimal & 76,6793 & 76,3911 & 73,4689 \\
\hline Mean_foptimal & 74,8873 & 74,3128 & 75,6092 \\
\hline Std_foptimal & 0,8544 & 1,0671 & 1,5463 \\
\hline
\end{tabular}

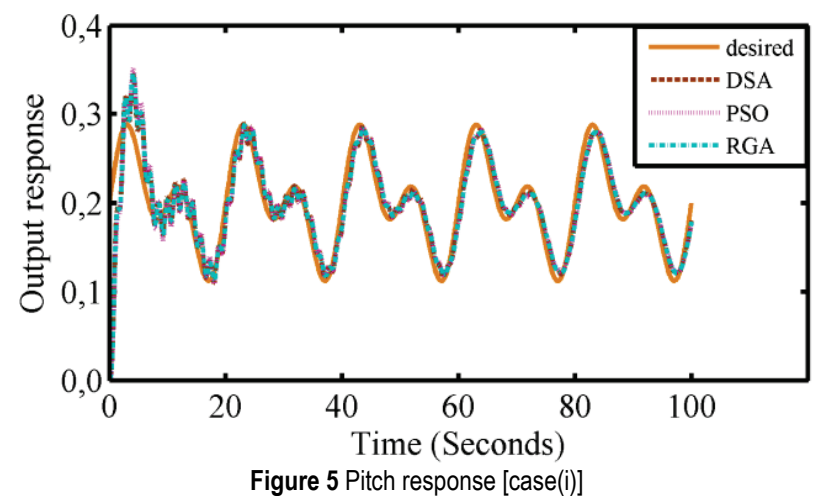

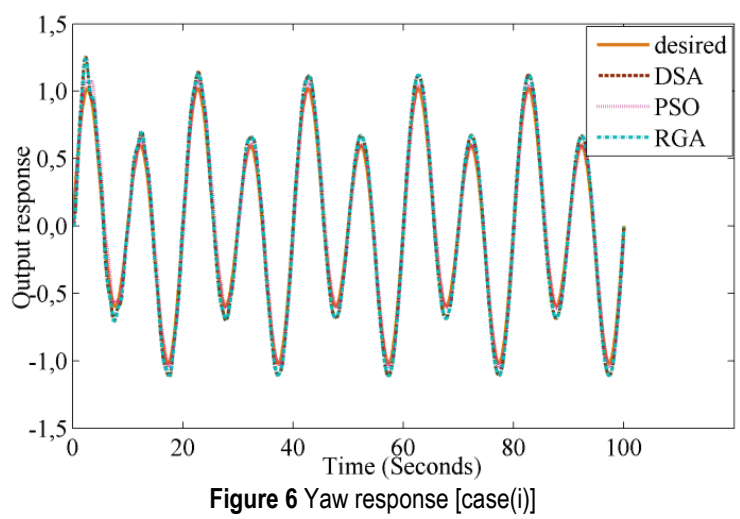

Fig. 4, Fig. 5 and Fig. 6, represent the convergence characteristics and output response of the pitch and yaw respectively for Nonlinear Combinational PID controller with fixed $\delta$ and $\alpha$ values. Its statistical performance is shown in Tab. 1. From the convergence characteristics (Fig. 4) PSO converges faster in the least optimal parameter compared to RGA and DSA and from Tab. 1 based on Best_optimal, Best Mean_optimal and Std_f optimal, PSO shows a better result than RGA and DSA. To track the desired input from Fig. 4 and Fig. 5 PSO shows better results.

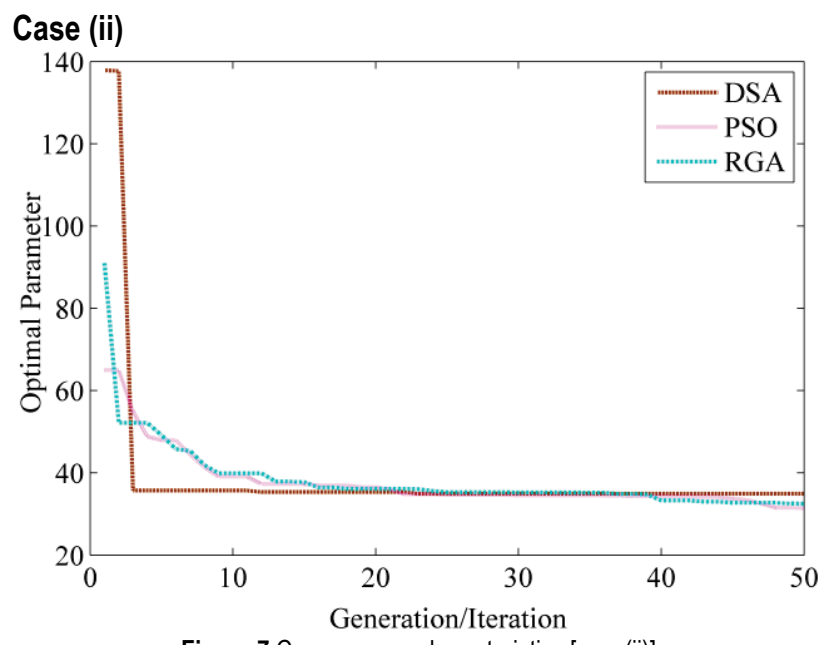

Figure 7 Convergence characteristics [case(ii)]

Table 2 Statistical performance of DSA, PSO and RGA for the Nonlinear PID controller [case(ii)]

\begin{tabular}{|c|c|c|c|}
\hline Evolutionary Algorithm & DSA & PSO & RGA \\
\hline Best_optimal & 34,8894 & 31,3880 & 32,4865 \\
\hline & 0,4999 & 0,5932 & 0,8298 \\
& 1,6870 & 1,8052 & 2,0696 \\
& 1,3084 & 2,4747 & 3,0290 \\
Best_optimal_parameter & 5,0000 & 4,7304 & 4,8934 \\
& 1,0048 & 0,0 & 3,4707 \\
& 4,9044 & 1,0283 & 0,9185 \\
& 0,0436 & 0,0525 & 0,0695 \\
& 0,1643 & 0,1127 & 0,1972 \\
\hline Worst_foptimal & 47,8734 & 34,3771 & 44,6754 \\
\hline Mean_foptimal & 39,1724 & 32,9550 & 35,4160 \\
\hline Std_foptimal & $2,952+4$ & 0,7252 & 2,8565 \\
\hline
\end{tabular}

Fig. 7, Fig. 8 and Fig. 9, represent the convergence characteristics and output response of the pitch and yaw respectively for Nonlinear PID controller with optimized $\delta$ and $\alpha$ values. Its statistical performance is shown in Tab. 2. From the convergence characteristics (Fig. 7) PSO converges faster in least optimal parameter compared to 
RGA and DSA and from Tab. 2 based on Best_optimal, Best Mean optimal and Std_foptimal, PSO shows better result than RGA and DSA. To track the desired input from Fig. 8 and Fig. 9 PSO shows better results.
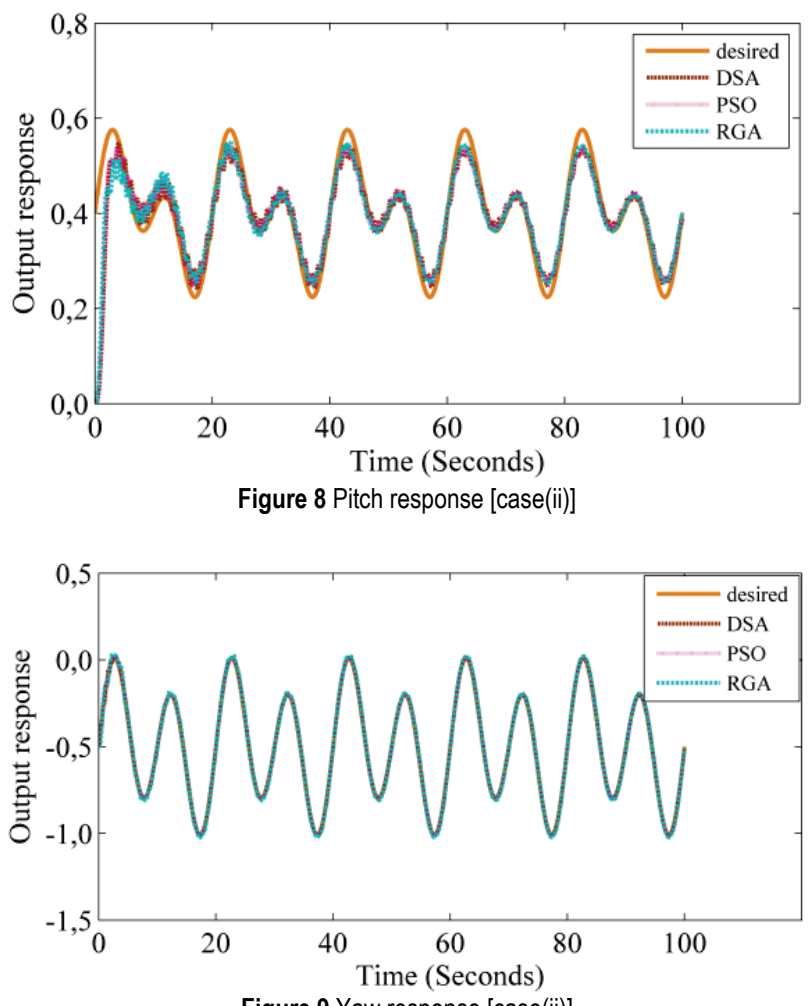

Figure 9 Yaw response [case(ii)]

\section{Case (iii)}

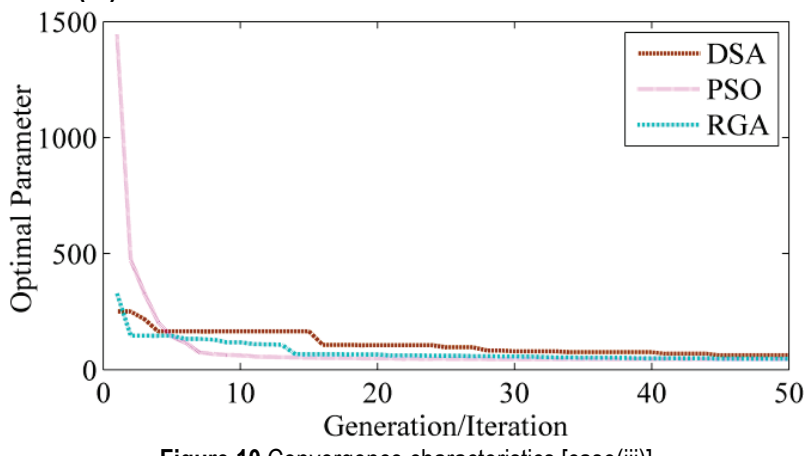

Figure 10 Convergence characteristics [case(iii)]

Table 3 Statistical performance of DSA, PSO and RGA for the Cross coupled Nonlinear PID controller [case(iii)]

\begin{tabular}{|c|c|c|c|}
\hline Evolutionary Algorithm & DSA & PSO & RGA \\
\hline Best_optimal & 62,1411 & 45,0429 & 47,1715 \\
\hline & 2,2512 & 2,1178 & 3,2600 \\
& 4,5158 & 5,0000 & 4,9556 \\
& 1,6616 & 2,7566 & 4,1462 \\
& 0,9816 & 0,0 & 0,0004 \\
& 0,0 & 1,5791 & 2,1516 \\
Best_optimal_parameter & 0,4612 & 1,5805 & 1,2512 \\
& 5,0000 & 5,0000 & 4,9969 \\
& 5,0000 & 0,5360 & 0,4854 \\
& 4,2252 & 5,0000 & 4,9857 \\
& 4,1605 & 5,0000 & 4,8877 \\
& 0,0 & 0,0 & 0,0325 \\
& 4,6827 & 5,0000 & 4,8760 \\
\hline Worst_foptimal & 100,8497 & 78,9464 & 81,4678 \\
\hline Mean_foptimal & 74,1571 & 53,2946 & 57,3072 \\
\hline Std_foptimal & 10,8884 & 5,0302 & 5,1475 \\
\hline
\end{tabular}
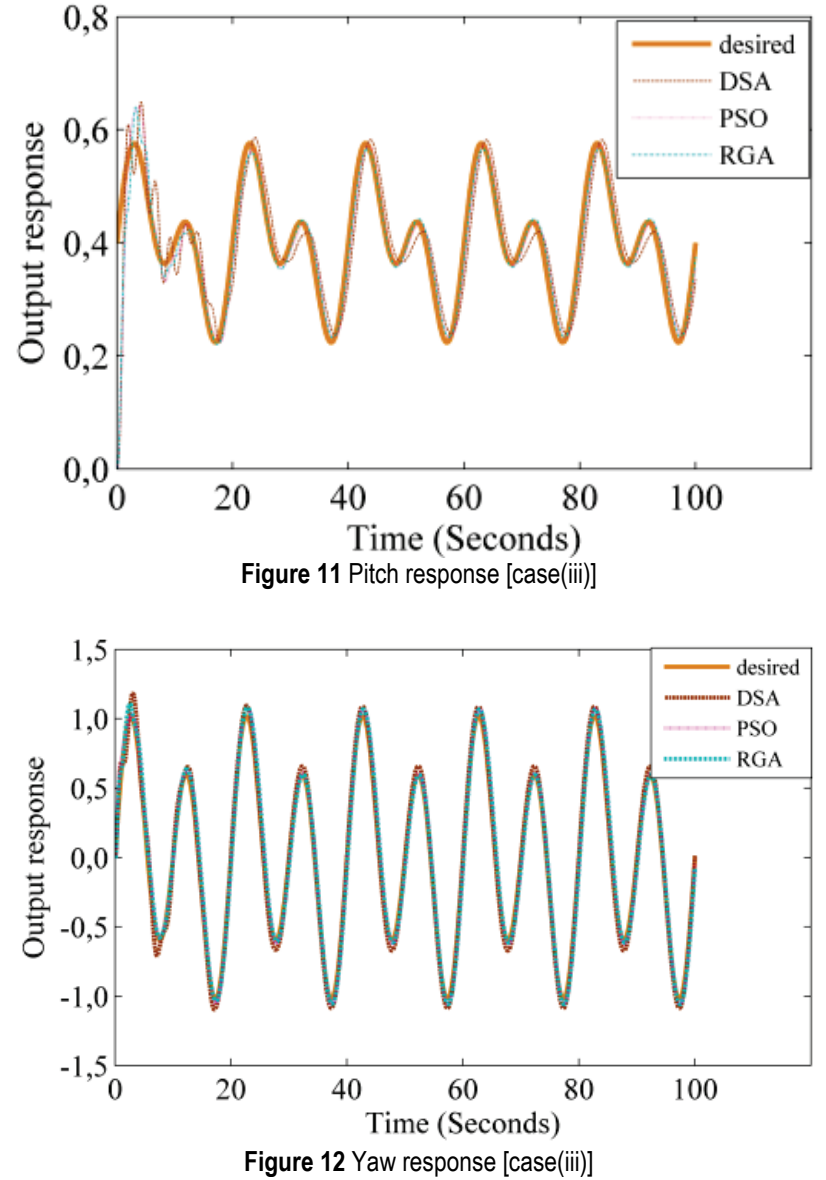

Case(iv)

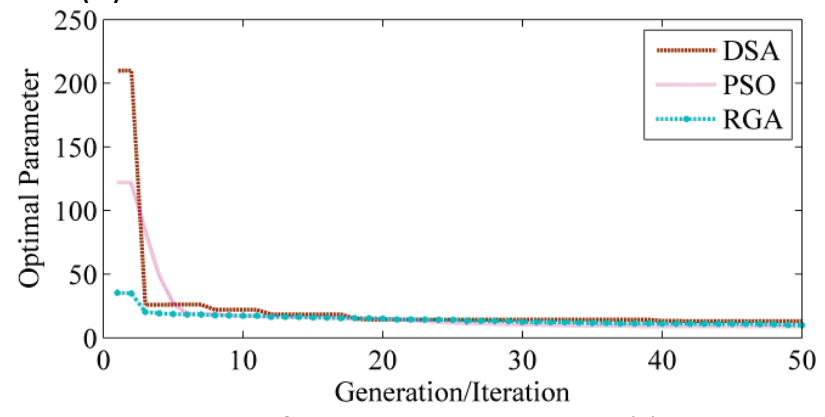

Figure 13 Convergence characteristics [case(iv)]

Table 4 Statistical performance of DSA, PSO and RGA for the Cross coupled Nonlinear PID controller [case(iv)]

\begin{tabular}{|c|c|c|c|}
\hline Evolutionary Algorithm & DSA & PSO & RGA \\
\hline Best_optimal & 13,1035 & 8,8527 & 9,9543 \\
\hline & 4,4476 & 5,0000 & 4,7874 \\
& 3,0211 & 5,0000 & 4,4084 \\
& 5,0000 & 2,1694 & 2,6645 \\
& 0,1467 & 0,0 & 0,0311 \\
& 4,1136 & 5,0000 & 1,3275 \\
& 4,0114 & 4,8458 & 3,2368 \\
Best_optimal_parameter & 4,7907 & 5,0000 & 4,9844 \\
& 2,4718 & 5,0000 & 2,6380 \\
& 4,2696 & 1,8612 & 1,2765 \\
& 1,9020 & 3,0662 & 3,5594 \\
& 0,0 & 0,0 & 0,2497 \\
& 0,0 & 0,0 & 0,0660 \\
& 0,0808 & 0,0586 & 0,0422 \\
& 0,1216 & 0,1000 & 0,1915 \\
\hline Worst_foptimal & 44,7482 & 21,4782 & 24,8712 \\
\hline Mean_foptimal & 22,5339 & 12,2519 & 14,4480 \\
\hline Std_foptimal & 7,8079 & 2,2223 & 2,7310 \\
\hline
\end{tabular}


Fig. 10, Fig. 11 and Fig. 12, represent the convergence characteristics and output response of the pitch and yaw respectively for Cross coupled Nonlinear PID controller with fixed $\delta$ and $\alpha$ values. Its statistical performance is shown in Tab. 3. From the convergence characteristics (Fig. 10) PSO converges faster in the least optimal parameter compared to RGA and DSA and from Tab. 3 based on Best_optimal, Best Mean_optimal and Std foptimal, PSO shows better result than RGA and DSA. To track the desired input from Fig. 11 and Fig. 12 PSO shows better results.

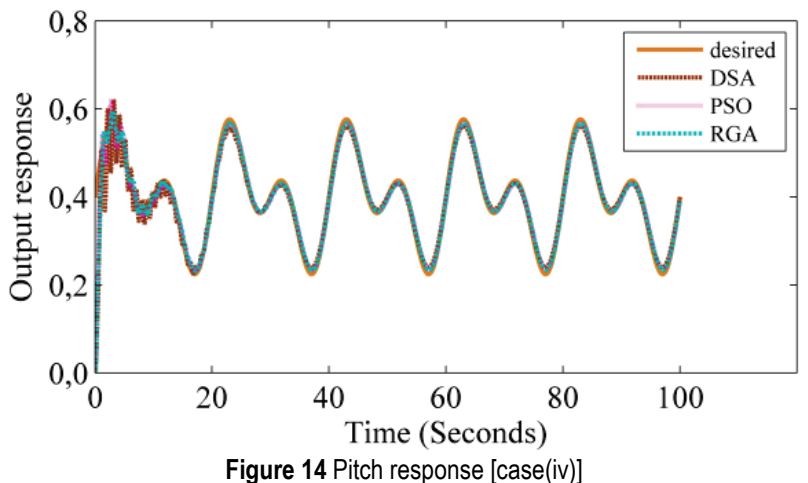

Fig. 13, Fig. 14 and Fig. 15, represent the convergence characteristics and output response of the pitch and yaw respectively for Cross coupled Nonlinear PID controller with optimized $\delta$ and $\alpha$ values. Its statistical performance is shown in Tab. 4. From the convergence characteristics (Fig. 13) PSO converges faster in least optimal parameter compared to RGA and DSA and from Tab. 4 based on Best_optimal, Best Mean_optimal and Std_f optimal, PSO shows better result than RGA and DSA. To track the desired input from Fig. 14 and Fig. 15 PSO shows better results.

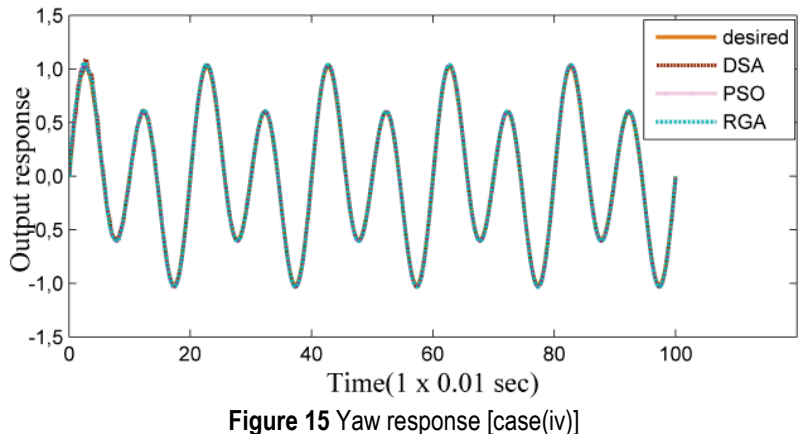

Considering the case (iv) Gravitational Search Algorithm (GSA) is applied and its statistical performance is given in Tab. 5 .

Table 5 The statistical performance of GSA [case(iv)]

\begin{tabular}{|l|r|}
\hline \multicolumn{1}{|c|}{ Statistical performance } & GSA \\
\hline Best optimal value & 25,3099 \\
\hline Worst optimal value & 80,3709 \\
\hline Mean optimal value & 31,9425 \\
\hline Standard deviation of optimal value & 14,9136 \\
\hline
\end{tabular}

Tab. 6 reveals that Cross coupled Nonlinear PID controller with optimized $\delta$ and $\alpha$ values shows better result comparatively. Also it is better than the results obtained from Tab. 5, statistical performance of GSA.
Table 6 The Best performance characteristics from case(i-iv)

\begin{tabular}{|c|c|c|c|c|}
\hline $\begin{array}{c}\text { Evolutionary } \\
\text { Algorithm }\end{array}$ & Case (i) & Case (ii) & Case (iii) & Case (iv) \\
\hline Best_optimal & 73,7812 & 31,3880 & 45,0429 & 8,8527 \\
\hline & 2,9922 & 0,5932 & 2,1178 & 5,0000 \\
& 5,0000 & 1,8052 & 5,0000 & 5,0000 \\
& 5,0000 & 2,4747 & 2,7566 & 2,1694 \\
& 5,0000 & 4,7304 & 0,0 & 0,0 \\
& 5,0000 & 0,0 & 1,5791 & 5,0000 \\
& 1,2626 & 1,0283 & 1,5805 & 4,8458 \\
Best_optimal_- & & 0,0525 & 5,0000 & 5,0000 \\
parameter & & 0,1127 & 0,5360 & 5,0000 \\
& & & 5,0000 & 1,8612 \\
& & & 5,0000 & 3,0662 \\
& & & 0,0 & 0,0 \\
& & & 5,0000 & 0,0 \\
& & & & 0,0586 \\
& & & & 0,1000 \\
\hline Worst_foptimal & 76,3911 & 34,3771 & 78,9464 & 21,4782 \\
\hline Mean_foptimal & 74,3128 & 32,9550 & 53,2946 & 12,2519 \\
\hline Std_foptimal & 1,0671 & 0,7252 & 5,0302 & 2,2223 \\
\hline
\end{tabular}

Our research shows the combination of elevation and azimuth i.e., vertical and horizontal which is better than the errors reported in previous researches [15-17], where the error values are reported separately (Vertical or Horizontal). Hence from the results it is observed that by optimizing the $\alpha$ and $\delta$, the results are better than in previous researches $[12,13]$ reported without optimizing $\alpha$ and $\delta$. PSO converges faster in the least optimal parameter in all the cases. Hence PSO shows better result with respect to output response and convergence characteristics.

\section{CONCLUSION}

The Evolutionary Computation is applied for tuning Nonlinear PID controller for TRMS by minimizing ISE value of the pitch and yaw response. The best, mean, worst and standard deviations of ISE value obtained from 20 independent trials are considered for comparing the performances of DSA, PSO, RGA and GSA. Simulation results reveal that the PSO performs better than other algorithm in terms of best value (least value compared to other algorithms) and better convergence characteristics. In future instead of Nonlinear PID controller, fractional PID controller with the influence of Nonlinear functions and evolutionary algorithms may be implied to tune TRMS, also model predictive controller may be utilized to enhance the tuning.

\section{Acknowledgments}

The authors gratefully acknowledge the Management of the National Engineering College, Kovilpatti-628503, Tamilnadu, India, for their continued support, encouragement and the extensive facilities provided to carry out this research work. They also gratefully acknowledge the financial support provided by All India Council for Technical Education for funding this project under Research Proposal Scheme. Ref: 20/AICTE/RIFD/RPS(POLICY-III)101/2012-13 Dated: 07/03/2013. 


\section{REFERENCES}

[1] Ang, K. H., Chang, G., \& Li, Y. (2005). PID control systems analysis, design and technology. IEEE Transactions on Control System Technology, 13(4), 559577. https://doi.org/10.1109/TCST.2005.847331

[2] Astrom, K. J. \& Hagglund, T. (1995). PID controllers: Theory, design, and tuning. $2^{\text {nd }} e d$. Instrument Society of America.

[3] Luyben, W. L. (1986). A Simple method for tuning SISO controllers in multivariable systems. Industrial \& Engineering Chemistry Process Design and Development, 25, 654-660. https://doi.org/10.1021/i200034a010

[4] Monica, T. J., Yu, C., \& Luyben, W. L. (1988). Improved multiloop single-input single- output (SISO) controllers for multivariable processes. Industrial and Engineering Chemistry Research, 27(6), 969-973. https://doi.org/10.1021/ie00078a013

[5] Wang, Q. G., Zou, B., Lee, T. H., \& Qiang, B. (1997) Auto-tuning of multivariable PID controllers from decentralized relay feedback. Automatica, 33(3), 319-330. https://doi.org/10.1016/S0005-1098(96)00177-X

[6] Chang, W. D. (2007). A multi-crossover genetic approach to multivariable PID controllers tuning. Expert System Applications, 33, 620-626. https://doi.org/10.1016/j.eswa.2006.06.003

[7] Zuo, W. (1995). Multivariable adaptive control for a space station using genetic algorithms. IEE Proceedings - Control Theory and Applications, 142(2), 81-87. https://doi.org/10.1049/ip-cta:19951734

[8] Su, C. T. \& Wong, J. T. (2007). Designing MIMO controller by neuro traveling particle swarm optimizer approach. Expert System Applications. 32, 848-855. https://doi.org/10.1016/j.eswa.2006.01.023

[9] Willjuice Iruthayarajan, M. \& Baskar, S. (2010). Covariance matrix adaptation evolution strategy based design of centralized PID controller. Expert System Applications, 37, 5775-5781. https://doi.org/10.1016/j.eswa.2010.02.031

[10] Civicioglu, P. (2012). Transforming geocentric Cartesian coordinates to geodetic coordinates by using differential search algorithm. Computers and Geoscience, 46, 229-247. https://doi.org/10.1016/j.cageo.2011.12.011

[11] Rashedi, E., Nezamabadi-pour, H., \& Saryazdi, S. (2009). GSA: A Gravitational Search Algorithm. Information Sciences, 179, 2232-2248. https://doi.org/10.1016/j.ins.2009.03.004

[12] Muthubalaji, S. \& Malathi, V. (2015). Multi-objective distribution feeder reconfiguration by considering energy not supplied with distributed generation. Tehnički vjesnik, 22(6). https://doi.org/10.17559/TV-20150118125107

[13] Tosun, S., Oztürk, A., Ertay, M. M., Yalçın, M. A., \& Zengin, A. (2015). An approach for designing passive power filters for industrial power Systems by using gravitational search algorithm. Tehnički vjesnik, 22(2), 343349. https://doi.org/10.17559/TV-20130822153221

[14] Han, J. (2009). From PID to Active Disturbance Rejection Control. IEEE Transactions on Industrial Electronics, 56(3), 900-906. https://doi.org/10.1109/TIE.2008.2011621

[15] Tan, K. K., Tong Heng Lee., \& Zhou, H. X. (2001). MicroPositioning of Linear-Piezoelectric Motors based on a Learning Nonlinear PID Controller. IEEE-ASME Transactions on Mechatronics, 6(4), 428-436. https://doi.org/10.1109/3516.974856

[16] Peng, X., Cheng, S., \& Wen, J. (2005). Application of Nonlinear PID Controller in Superconducting Magnetic Energy Storage. International Journal of Control, Automation and Systems, 3(2)(special edition), 296-301.
[17] Tao, C. W., Taur, J. S., \& Chen, Y. C. (2010). Design of a parallel distributed fuzzy LQR controller for the twin rotor multi-input multi-output system. Fuzzy Sets and Systems, 161, 2081-2103. https://doi.org/10.1016/j.fss.2009.12.007

[18] Taskin, Y. (2014). Improving pitch and yaw motion control of twin rotor MIMO system. Journal of Vibroengineering, 16(4), 1650-1659.

[19] Jih-Gau Juang., Wen-Kai Liu., \& Ren-Wei Lin. (2011). A hybrid intelligent controller for a twin rotor MIMO system and its hardware implementation. ISA Transactions, 50, 609-619. https://doi.org/10.1016/j.isatra.2011.06.006

[20] Juang, Jih-Gau. \& Tu, Kai-Ti. (2013). Design and Realization of a Hybrid Intelligent Controller for a Twin Rotor MIMO System. Journal of Marine Science and Technology - Japan, 21(3), 333-341

[21] Rahideh, A., Bajodah, A. H., \& Shaheed, M. H. (2012). Real time adaptive nonlinear model inversion control of a twin rotor MIMO system using neural networks. Engineering Applications of Artificial Intelligence, 25, 1289-1297. https://doi.org/10.1016/j.engappai.2011.12.006

[22] Rotondo, D., Nejjari, F., \& Puig, V. (2013). Quasi-LPV modeling, identification and control of a twin rotor MIMO system. Control Engineering Practice. 21, 829-846. https://doi.org/10.1016/j.conengprac.2013.02.004

[23] Hashim, H. A. \& Abido, M. A. (2015). Fuzzy Controller Design Using Evolutionary Techniques for Twin Rotor MIMO System: A Comparative Study. Hindawi Publishing Corporation, Computational Intelligence and Neuroscience. https://doi.org/10.1155/2015/704301

[24] Ates, A., Alagoz, B. B., \& Yeroglu, C. (2017). MasterSlave Stochastic Optimization for Model-Free Controller Tuning. Iranian Journal of Science and Technology, Transactions of Electrical Engineering, 41(2), 153-163. https://doi.org/10.1007/s40998-017-0029-1

[25] Waleed Abdulwahhab, O. \& Hadi Abbas, N. (2017). A New method to tune a Fractional-Order PID controller for Twin Rotor Aerodynamic System. Arabian Journal for Science and Engineering, 42(12), 5179-5189. https://doi.org/10.1007/s13369-017-2629-5

[26] Ahammad Shaik, F., Purwar, S., \& Pratap, B. (2011). Realtime implementation of Chebyshev neural network observer for twin rotor control system. Expert Systems with Applications, 38, 13043-13049. https://doi.org/10.1016/j.eswa.2011.04.107

\section{Contact information:}

J. SIVADASAN, Assistant Professor (Sr, Grade), EEE Department National Engineering College

K. R. Nagar, Kovilpatti, Tamilnadu, India-628503 sivadasan@nec.edu.in

M. WILLJUICE IRUTHAYARAJAN, Professor \& Head, EEE Department National Engineering College

K. R. Nagar, Kovilpatti, Tamilnadu, India-628503

m.willjuice@gmail.com 